\title{
Análise Comparativa de Modelos Analíticos para a Predição do Tempo de Vida de Baterias sob o mesmo Cenário de Simulação considerando Correntes de Descargas Variáveis
}

Julia Giehl Zart ${ }^{1}$

Douglas Joziel Bitencourt Freitas ${ }^{2}$

Airam Teresa Zago Romcy Sausen ${ }^{3}$

Paulo Sérgio Sausen ${ }^{4}$

Programa de Pós-Graduação Stricto Sensu em Modelagem Matemática, UNIJUÍ, Ijuí, RS

Resumo. Neste artigo é apresentada uma análise comparativa entre os modelos analíticos, Linear, Lei de Peukert, Lei de Peukert Estendida, KiBaM e modelo de difusão de Rakhmatov e Vrudhula (RV), utilizados para predizer o tempo de vida de baterias que alimentam dispositivos móveis. Tais modelos foram analisados e validados a partir de ensaios reais, objetivando verificar qual o modelo analítico é mais acurado para a predição do tempo de vida de baterias de Lítio Íon Polímero (LiPo), considerando o mesmo cenário de simulação, e utilizando perfis de descarga variáveis, baseadas em um conjunto de operações rotineiras realizadas em um telefone celular do tipo smartphone. A partir dos resultados, verificou-se que o modelo analítico RV é o mais acurado no que se refere à predição do tempo de vida de baterias do tipo LiPo.

Palavras-chave. Modelagem Matemática, Modelos Analíticos, Baterias de LiPo, Tempo de Vida

\section{Introdução}

Em meados dos anos 90, os telefones celulares apenas permitiam a troca de mensagens curtas de texto e a realização de chamadas de voz. No entanto, este cenário mudou rapidamente, pois viu-se as potencialidades desses dispositivos em agregar inúmeras outras funcionalidades. Hoje, além das operações básicas, um smartphone permite ao usuário executar diversos aplicativos e manter-se constantemente conectado à internet, dentre outras opções. Assim, com o aumento das funcionalidades disponíveis, e com as telas coloridas que os mesmos possuem, ocorreu um significativo aumento do consumo de energia, o que resultou em um menor tempo de vida ${ }^{5}$ das baterias.

\footnotetext{
${ }^{1}$ julia_zarte@hotmail.com

22douglasjoziel@outlook.com

3 airam@unijui.edu.br

${ }^{4}$ sausen@unijui.edu.br

${ }^{5}$ Neste artigo, o tempo de vida é definido com a duração de uma única descarga completa da baterias, estando esta inicialmente carregada por completo.
} 
Desta maneira, com o intuito de aperfeiçoar os sistemas de gerenciamento de energia das baterias que alimentam os dispositivos móveis, tornando mais longos os períodos sem a necessidade de carregamento externo, é de vital importância possuir um método capaz de predizer tanto o tempo de vida da bateria, quanto o comportamento dinâmico do processo de descarga. Uma maneira de realizar essa predição é através de modelos matemáticos que simulam o processo de descarga das baterias a partir de suas características físicas reais, ou de um conjunto de dados obtidos de ensaios experimentais. Inserido nesse contexto, o Grupo de Automação Industrial e Controle (GAIC/UNIJUÍ), realiza estudos objetivando verificar qual é o modelo matemático mais apropriado para predizer o tempo de vida de baterias utilizadas em dispositivos móveis.

O principal objetivo deste trabalho é realizar uma análise comparativa entre os modelos analíticos - Linear, Lei de Peukert, Lei de Peukert Estendida, KiBaM, e RV - considerando o mesmo cenário de simulação e perfis de descargas variáveis baseadas nas principais funcionalidades de um smartphone, a fim de verificar qual é o modelo analítico que realiza a predição do tempo de vida de baterias de forma mais acurada. As simulações dos modelos são realizadas no software de computação algébrica e numérica MatLab e a validação dos modelos é efetuada comparando os resultados simulados com os dados experimentais obtidos de uma plataforma de testes.

O presente artigo está organizado como segue. Na Seção 2 são descritos os modelos analíticos utilizados neste estudo, dando ênfase as suas equações. Na Seção 3 é apresentada de modo breve a metodologia adotada para a coleta dos dados experimentais, assim como, a estimação dos parâmetros dos modelos, utilizando o método dos Mínimos Quadrados (MQ). Na Seção 4 são discutidos os resultados das simulações, através de uma análise comparativa entre todos os modelos, com o intuito de verificar qual é o modelo mais acurado em questão de predição do tempo de vida de baterias. E, por fim, na Seção 5, são apresentadas as considerações finais e perspectivas para os trabalhos futuros.

\section{Modelagem Matemática}

Nesta seção são descritos os cinco modelos matemáticos analíticos utilizados neste estudo para a predição do tempo de vida de baterias do tipo LiPo que alimentam dispositivos móveis, considerando o mesmo cenário de simulação. Os modelos analíticos foram escolhidos porque utilizam um conjunto reduzido de equações, possuem menos parâmetros para serem estimados quando comparado aos demais modelos matemáticos, e apresentam simulação computacionalmente versátil e eficiente.

\subsection{Modelo Linear}

O modelo Linear $[2,6]$ é considerado pela literatura técnica o mais simples dos modelos analíticos e de fácil implementação. Na prática, durante o processo de descarga, a bateria apresenta efeitos não lineares, como o efeito da taxa de capacidade e o efeito de recuperação, que interferem diretamente no tempo de vida da mesma [2]. Este modelo não considera tais efeitos, visto que a bateria é tratada como um recipiente linear de corrente.

Em perfis de descargas variáveis, o tempo total de descarga é dividido em vários 
períodos aproximados, de correntes constantes por partes, conforme a Figura 1. Na qual observa-se que do tempo $t_{0}$ ao tempo $t_{1}$, a corrente que age no sistema é a corrente inicial, representada por $I_{0}$. Já durante o período de tempo $t_{1}$ ao tempo $t_{2}$, a corrente $I_{1}$ atua no sistema. Desta forma, as correntes são alternadas até que a bateria não consiga mais fornecer energia ao sistema (i.e., nível de cutoff).

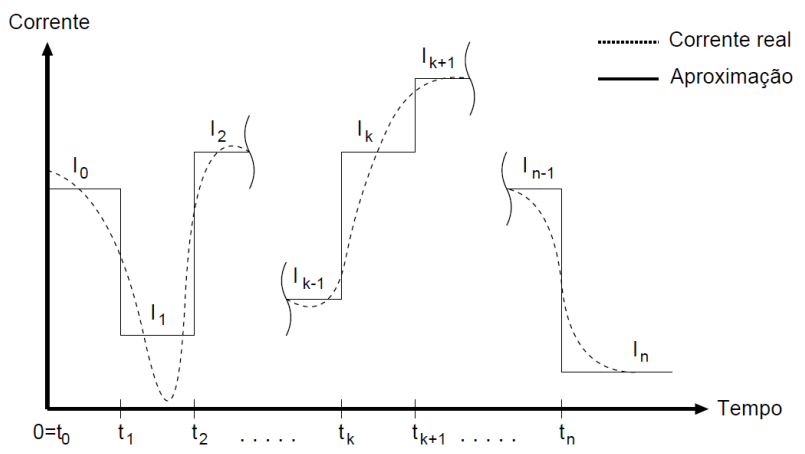

Figura 1: Degraus representativos de perfil de descarga com correntes variáveis [4].

Logo, para correntes de descargas variáveis, a corrente de descarga $(I)$ é a média das correntes ao longo do tempo no intervalo de $[0, L][4]$. Assim o tempo de vida de uma bateria, a partir do modelo Linear, é dado pela seguinte equação

$$
L=\frac{C_{i}}{\left[\frac{\sum_{k=1}^{n} I_{k-1}\left(t_{k}-t_{k-1}\right)}{L}\right]}
$$

onde: $I_{k}$ é a corrente de descarga, $t_{k}$ é o tempo inicial da corrente de descarga, $t_{k-1}$ é o tempo final da corrente utilizada e, $C_{i}$ é o único parâmetro a determinar, que está relacionado a capacidade inicial da bateria.

\subsection{Lei de Peukert}

A Lei de Peukert é um modelo simples e intuitivo que considera somente uma das características não lineares, o efeito da taxa de capacidade [2]. Conforme a Lei de Peukert [4], o tempo de vida $(L)$ de uma bateria para descargas variáveis é dado pela expressão

$$
L=\frac{a}{\left[\frac{\sum_{k=1}^{n} I_{k-1}\left(t_{k}-t_{k-1}\right)}{L}\right]^{b}}
$$

onde: $a$ é um valor próximo ao da capacidade da bateria e, $b$ é o coeficiente de Peukert, sendo os parâmetros que precisam ser estimados a partir de dados experimentais, e variam conforme o tipo de bateria utilizada.

\subsection{Lei de Peukert Estendida}

Visando diminuir o erro médio apresentado pela Lei de Peukert, Freitas [1] desenvolveu a Lei de Peukert Estendida, na qual o tempo de vida de uma bateria considerando correntes de descargas variáveis é descrita pela equação 


$$
L=\left(\frac{\left[\frac{\sum_{k=1}^{n} I_{k-1}\left(t_{k}-t_{k-1}\right)}{L}\right]-\sqrt{\left[\frac{\sum_{k=1}^{n} I_{k-1}\left(t_{k}-t_{k-1}\right)}{L}\right]^{2}-4 C_{1} C_{2}}}{2 C_{1}}\right)^{b},
$$

onde: $C_{1}$ é o coeficiente de ajuste não linear, $C_{2}$ é a capacidade análoga a capacidade física da bateria e, $b$ é o coeficiente de Peukert, sendo os parâmetros a determinar a partir de um conjunto de dados experimentais.

\subsection{Modelo KiBaM}

O modelo analítico cinético KiBaM é capaz de capturar os dois efeitos não lineares presentes no processo de descarga (i.e., efeito de recuperação e efeito da taxa de capacidade), assim como utiliza a cinética do processo químico que ocorre no interior da bateria [2]. Este modelo distribui a carga da bateria em duas fontes: a fonte de carga disponível $\left(y_{1}\right)$ e a fonte de carga limitada $\left(y_{2}\right)$. No momento em que é aplicada uma corrente de descarga na bateria, a fonte de carga disponível, fornece elétrons para a corrente $i(t)$, desta forma, a altura desta fonte diminui, o que faz com que a diferença entre as alturas $h_{1}(t)$ e $h_{2}(t)$ aumente. Contudo, quando a corrente de descarga torna-se nula, a carga da fonte limitada flui para a fonte de carga disponível até que as alturas $h_{1}(t)$ e $h_{2}(t)$ se igualem novamente $[2,3]$. As equações que descrevem este modelo são dadas por

$$
y_{1}(t)=y_{1}(0) e^{-k^{\prime} t}+\frac{\left(y_{0} k^{\prime} c-I\right)\left(1-e^{-k^{\prime} t}\right)}{k^{\prime}}-\frac{I c\left(k^{\prime} t-1+e^{-k^{\prime} t}\right)}{k^{\prime}}
$$

e

$$
y_{2}(t)=y_{2}(0) e^{-k^{\prime} t}+y_{0}(1-c)\left(1-e^{k^{\prime} t}\right)-\frac{I(1-c)\left[k^{\prime} t-1+e^{-k^{\prime} t}\right]}{k^{\prime}},
$$

onde: $c$ é uma fração da capacidade total, $k^{\prime}$ é a razão do fluxo de carga entre as duas fontes e suas respectivas capacidades e, $y_{0}$ é a quantidade total de carga da bateria. A bateria é considerada descarregada quando não há mais carga na fonte de carga disponível $\left(y_{1}=0\right)$.

\subsection{Modelo de Rakhmatov e Vrudhula}

O modelo RV captura tanto o efeito da taxa de capacidade, como o efeito de recuperação, sendo considerado de alta acurácia pela literatura técnica [4]. É baseado na difusão de íons no eletrólito e descreve a evolução unidimensional da concentração das espécies eletroativas através de um sistema de Equações Diferenciais Parciais (EDPs). A solução deste modelo para descargas variáveis, expressa uma relação entre o tempo de vida $L$ da bateria e a carga variável $i(t)$, que é aproximada por uma função de $n$ degraus no intervalo $[0, L]$, dada por

$$
\alpha=\sum_{k=1}^{n} 2 I_{k-1} A\left(L, t_{k}, t_{k-1}, \beta\right)
$$


onde:

$$
\begin{aligned}
A\left(L, t_{k}, t_{k-1}, \beta\right)= & \sqrt{L-t_{k-1}}\left[1+2 \sum_{m=1}^{10} e \frac{-\beta^{2} m^{2}}{L-t_{k-1}}-\frac{\pi e \frac{-\beta^{2} m^{2}}{L-t_{k-1}}}{\pi-1+\sqrt{1+\pi \frac{L-t_{k-1}}{\beta^{2} m^{2}}}}\right] \\
& -\sqrt{L-t_{k}}\left[1+2 \sum_{m=1}^{10}\left(e \frac{-\beta^{2} m^{2}}{L-t_{k}}-\frac{\pi e \frac{-\beta^{2} m^{2}}{L-t_{k}}}{\pi-1+\sqrt{1+\pi \frac{L-t_{k}}{\beta^{2} m^{2}}}}\right)\right]
\end{aligned}
$$

$\alpha$ é o parâmetro que está relacionado com a capacidade da bateria e $\beta$ é o parâmetro que está relacionado ao comportamento não linear da bateria.

\section{Dados Experimentais e Estimação dos Parâmetros}

$\mathrm{Na}$ descrição dos modelos analíticos apresentados na seção anterior, observa-se que os mesmos possuem parâmetros empíricos que necessitam ser estimados. O processo de estimação de parâmetros de um modelo matemático ocorre a partir do conhecimento de um conjunto de dados experimentais e da definição de uma metodologia para a estimação dos parâmetros.

Nos ensaios laboratoriais foram utilizadas 8 baterias novas do tipo LiPo, modelo PL383562-2C. Para a estimação dos parâmetros, foram empregados 4 perfis de descargas constantes, variando de $75 \mathrm{~mA}$ a $675 \mathrm{~mA}$, com intervalo entre os perfis de $200 \mathrm{~mA}$. Cada perfil gerou, de modo individual, um tempo de descarga para cada bateria. A partir desses ensaios, foi calculado o tempo de vida médio para cada perfil, que é utilizado nas simulações computacionais. Ressalta-se que seguindo a metodologia adotada em [4], perfis de descargas constantes são suficientes para a estimação dos parâmetros dos modelos que descrevem o comportamento das baterias, tanto para correntes de descargas constantes, quanto para correntes de descargas variáveis.

A estimação dos parâmetros, para os cinco modelos, foi realizada através do método dos MQ, que é um método de otimização matemática que procura encontrar o valor ótimo dos parâmetros para um determinado conjunto de dados, através da minimização da soma dos quadrados das diferenças entre o valor estimado e os dados experimentais (i.e., resíduos). Os parâmetros foram obtidos com o auxílio da ferramenta computacional MatLab, através da função de otimização "Isqnonlin", que possui internamente a rotina dos MQ para problemas de otimização não linear. O parâmetro encontrado para o modelo Linear foi $C_{i}=45486$. Para o modelo Lei de Peukert, foram obtidos os parâmetros $a=46692 ; \mathrm{e}, b=1,0059$. Já para a Lei de Peukert Estendida, os parâmetros encontrados foram $C_{1}=-0,0036 ; C_{2}=40651 ; \mathrm{e}, b=1,0222$. Para o modelo KiBaM, os parâmetros encontrados foram $y_{0}=45801 ; c=6,9594.10^{-8} ; \mathrm{e}, k^{\prime}=7364100$. E para o modelo RV, os parâmetros foram $\alpha=29867$; e, $\beta=2,7614$.

Para a validação dos modelos, foram considerados 8 perfis de descargas variáveis (cf. Tabela 1), definidos a partir de correntes de descargas que representam as funções aplicadas em um smarthphone, o que aproxima a simulação de um processo real. Diferentemente das descargas contantes, estes oito perfis possuem variação ao longo do tempo, de forma que 
os efeitos não lineares ficam mais presentes, e como consequência, o processo de descarga torna-se mais próximo ao perfil utilizado pelo usuário em um smartphone.

Tabela 1: Ciclo dos perfis de correntes de descarga variáveis.

\begin{tabular}{ccc}
\hline Perfil & Correntes (mA) & Tempo $(\mathbf{m i n})$ \\
\hline P1 & $100-10-150-10-100-10-200$ & $5-5-5-5-5-5-10$ \\
P2 & $170-270-10-140-230-10-270$ & $5-20-30-10-20-10-30$ \\
P3 & $270-10-120-170-10-270-170$ & $5-10-10-15-10-15-5$ \\
P4 & $250-400-50-200-550$ & $10-10-5-15-10$ \\
P5 & $750-450-200-150-250-100$ & $5-10-10-5-5-10$ \\
P6 & $100-200-300-400-500-600-700$ & $10-10-10-10-10-10-10$ \\
P7 & $700-600-500-400-300-200-100$ & $10-10-10-10-10-10-10$ \\
P8 & $200-10-300-10-200-10-400$ & $2,5-5-2,5-5-2,5-5-5$ \\
\hline
\end{tabular}

Ressalta-se que, em todos os perfis descritos, as correntes se repetem continuamente até que a bateria atinja o nível de cutoff. Através da plataforma de testes, foi obtido um conjunto de valores para o tempo de vida médio de cada perfil, apresentado na Tabela 2 , sendo este conjunto utilizado nas simulações computacionais no MatLab para a validação e análise comparativa dos modelos analíticos. Destaca-se ainda que os modelos analíticos analisados nesta pesquisa, possuem o mesmo cenário de simulação. Ou seja, para a simulação, é utilizado o mesmo conjunto de dados experimentais obtidos nos processos de descarga de baterias de LiPo, a metodologia adotada para a estimação dos parâmetros é a mesma, assim como, a estrutura dos algoritmos no MatLab segue o mesmo padrão.

\section{Análise dos Resultados}

A partir dos parâmetros obtidos os modelos analíticos são implementados e simulados na ferramenta computacional MatLab a fim de realizar a sua validação. Os resultados encontrados nas simulações foram comparados com um conjunto de dados experimentais obtidos na plataforma de testes, conforme apresentado na Tabela 2, além de ser realizada uma análise comparativa entre os modelos estudados, com o objetivo de verificar qual é o modelo analítico mais acurado para predizer o tempo de vida de uma bateria de LiPo quando dada as mesmas condições de comparação (i.e., mesmo cenário de simulação).

Tabela 2: Análise comparativa entre os modelos analíticos.

\begin{tabular}{|c|c|c|c|c|c|c|c|c|c|c|c|}
\hline \multicolumn{2}{|c|}{$\begin{array}{c}\text { Dados } \\
\text { Experimentais }\end{array}$} & \multicolumn{2}{|c|}{ Linear } & \multicolumn{2}{|c|}{ Peukert } & \multicolumn{2}{|c|}{$\begin{array}{c}\text { Peukert } \\
\text { Estendido }\end{array}$} & \multicolumn{2}{|c|}{ KiBaM } & \multicolumn{2}{|c|}{ RV } \\
\hline Perfil & $\mathrm{L}(\min )$ & $\mathrm{L}(\min )$ & Erro (\%) & $\mathrm{L}(\min )$ & Erro (\%) & $\mathrm{L}(\min )$ & Erro (\%) & $\mathrm{L}(\min )$ & Erro (\%) & $\mathrm{L}(\min )$ & Erro (\%) \\
\hline $\mathrm{P} 1$ & 479,72 & 473,50 & 1,30 & 473,40 & 1,32 & 474,80 & 1,03 & 473,10 & 1,38 & 477,90 & 0,38 \\
\hline P2 & 284,95 & 266,70 & 6,40 & 266,00 & 6,65 & 267,30 & 6,19 & 265,90 & 6,69 & 266,90 & 6,33 \\
\hline P3 & 322,02 & 317,00 & 1,56 & 316,20 & 1,81 & 318,60 & 1,06 & 316,90 & 1,59 & 319,40 & 0,81 \\
\hline P4 & 149,39 & 149,60 & 0,14 & 149,00 & 0,26 & 149,10 & 0,19 & 148,20 & 0,80 & 148,20 & 0,80 \\
\hline P5 & 141,76 & 144,50 & 1,93 & 143,70 & 1,37 & 143,90 & 1,51 & 143,20 & 1,02 & 143,40 & 1,16 \\
\hline P6 & 126,63 & 124,20 & 1,92 & 123,60 & 2,39 & 123,50 & 2,47 & 122,80 & 3,02 & 122,80 & 3,02 \\
\hline P7 & 98,52 & 99,00 & 0,49 & 98,10 & 0,42 & 97,80 & 0,73 & 97,70 & 0,83 & 97,80 & 0,73 \\
\hline $\mathrm{P} 8$ & 324,17 & 326,80 & 0,81 & 326,50 & 0,72 & 327,40 & 1,00 & 325,60 & 0,44 & 326,60 & 0,75 \\
\hline & ro Médio & (\%) & 1,82 & & 1,87 & & 1,77 & & 1,97 & & 1,75 \\
\hline
\end{tabular}

Os modelos predizem satisfatoriamente os dados experimentais, podendo ser empregados na predição do tempo de vida de baterias de LiPo para descargas variáveis, pois o erro médio de todos os modelos foi inferior a $2 \%$, e a literatura considera valores inferiores a $5 \%$ como de boa precisão [5]. Conforme pode ser observado, considerando o erro médio de cada modelo, o modelo analítico mais acurado desta comparação é o RV, com um erro 
médio de 1,75\%. Posteriormente, tem-se a Lei de Peukert Estendida, que obteve um erro médio de $1,77 \%$. Na sequência, o terceiro modelo mais acurado considerando descargas variáveis e o mesmo cenário de simulação é o Linear, com um erro médio de 1,82\%. Dando continuidade, tem-se a Lei de Peukert, com 1,87\% de erro médio. E por fim, o modelo menos acurado desta comparação, o modelo KiBaM, que obteve um erro médio de 1,97\% quanto comparado aos dados experimentais.

\section{Conclusões}

Através da análise do erro médio de cada modelo, conclui-se que o modelo RV é o mais indicado para predizer o tempo de vida de baterias considerando descargas variáveis, confirmando os estudos que dizem que este modelo é de alta acurácia. Na sequência, com uma diferença de acurácia mínima (i.e., 0,02\%), tem-se então, a Lei de Peukert Estendida, que possui equações mais simples quando comparado ao modelo RV. Como trabalhos futuros, sugere-se investigar a classificação de acurácia dos modelos encontrados neste estudo, incluindo uma análise mais detalhada dos resultados, pois acreditava-se que o modelo KiBaM apresentaria um resultado de erro médio semelhante ao modelo RV, em decorrência destes dois capturaram os efeitos não lineares presentes no processo de descarga das baterias. Além disso, sugere-se ampliar essa análise comparativa utilizando o mesmo cenário de experimentação e simulação para as demais classes de modelos de bateria, uma vez que o presente estudo limitou-se aos modelos analíticos.

\section{Referências}

[1] D. J. B. Freitas, P. S. Sausen, A. T. Z. R. Sausen e M. M. P. Reimbold. Extensão da Lei de Peukert aplicada à predicação do tempo de vida de baterias de lithium-ion polymer. In DINCON'15, Natal, Rio Grande do Norte, 2015.

[2] M. R. Jorgerden and B. Haverkort. Battery modeling. Technical report, Faculty Electrical Engineering, jan. 2008.

[3] J. Manwell and J. McGowan. Lead acid battery storage model for hybrid energy systems. Solar Energy, vol. 50, pp. 399-405, mai. 1993.

[4] D. Rakhmatov and S. Vrudhula. An analytical high-level battery model for use in energy management of portable electronic systems. In Proceedings of the International Conference on Computer Aided Design (ICCAD '01), Piscataway, 2001. IEEE Press.

[5] M. F. Triola, Introdução à Estatística. LTC, Rio de Janeiro, 1999.

[6] J. G. Zart, Análise Comparativa de Modelos Analíticos sob o mesmo Cenário de Simulação para Perfis de Descargas Variáveis considerando as Funções Desempenhadas por um Smartphone, Dissertação de Mestrado, Unijuí, Ijuí-RS, 2017. 